\title{
Serum proteomics analysis and comparisons using iTRAQ in the progression of hepatitis $B$
}

\author{
LIANG PENG $^{1^{*}}$, JING LIU $^{1 *}$, YANG-MEI LI $^{2 *}$, ZHAN-LIAN HUANG $^{1}$, PEI-PEI WANG ${ }^{1}$, \\ YU-RONG GU ${ }^{1}$, YU-BAO ZHENG ${ }^{1}$ and ZHI-LIANG GAO ${ }^{1}$ \\ Departments of ${ }^{1}$ Infectious Diseases and ${ }^{2}$ Traditional Chinese Medicine, Third Affiliated Hospital \\ of Sun Yat-sen University, Guangzhou, Guangdong 510630, P.R. China
}

Received May 15, 2013; Accepted September 12, 2013

DOI: $10.3892 /$ etm.2013.1310

\begin{abstract}
The aim of this study was to analyze the changes in serum protein levels in the progression of hepatitis B using isobaric tags for relative and absolute quantitation (iTRAQ) analysis, in addition to comparing the serum protein levels of patients with chronic hepatitis $\mathrm{B}(\mathrm{CHB})$, patients with hepatitis B virus-induced acute-on-chronic liver failure (HBV-induced ACLF) and normal individuals. Protein analysis was performed on 15 serum samples using iTRAQ. The study population included healthy controls $(n=5)$, patients with $\mathrm{CHB}$ $(n=5)$ and patients with HBV-induced ACLF $(n=5)$. Western blotting was used to verify the results in an additional nine serum samples from healthy controls, patients with $\mathrm{CHB}$ and patients with $\mathrm{HBV}$-induced ACLF ( $\mathrm{n}=3$, respectively). Using iTRAQ analysis, 16 different serum proteins with $\geq 1.5$-fold differences in expression levels were identified in the patients with CHB and ACLF compared with the healthy controls. Five of those proteins, $\mathrm{C}$-reactive protein precursor, hemoglobin $\beta$ chain variant $\mathrm{Hb} \mathrm{S}-\mathrm{Wake}$, apolipoprotein $\mathrm{J}$ precursor, platelet factor 4 precursor and vitronectin, which demonstrated the greatest differences in their expression levels and the most significant correlation with liver diseases, were subsequently verified using western blotting. The western blotting results were consistent with the results from the iTRAQ. Two of the five proteins are not classified by biological process, and the biological functions of all the proteins in HBV-induced ACLF remain unclear. This preliminary study demonstrated that a correlation between the expression of various serum proteins
\end{abstract}

Correspondence to: Professor Zhi-Liang Gao, Department of Infectious Diseases, Third Affiliated Hospital of Sun Yat-sen University, 600 Tianhe Road, Guangzhou, Guangdong 510630, P.R. China

E-mail: zhilianggao@21cn.com

${ }^{*}$ Contributed equally

Key words: serum, proteomics, isobaric tags for relative and absolute quantitation, hepatitis B and the different pathogenetic conditions induced by HBV may exist. The analysis of a larger number of samples is required to identify potential protein biomarkers that may be involved in the pathogenesis and progression of hepatitis B.

\section{Introduction}

In China, the hepatitis B surface antigen (HBsAg) seropositive rate for the general population (between 1 and 59 years of age) is $7.18 \%$ (1). Globally, there are 93 million individuals with hepatitis B virus (HBV) infections, 20 million of which are chronic (2). Hepatitis B is the most common risk factor for liver cirrhosis and hepatocellular carcinoma (HCC), and the mortality of $\mathrm{HBV}$-induced acute-on-chronic liver failure (ACLF) may exceed 60\%. HBV infections are not easy to cure, and the mechanisms of the virus are unclear, particularly with regard to protein expression and regulation function in the pathogenic process.

Proteomics analysis is a powerful technology used in a myriad of studies, including those focused on liver diseases (3-7). Isobaric tags for relative and absolute quantitation (iTRAQ), as a quantitative method, is a common tool in proteomics and has been suggested to be as sensitive as (or more sensitive than) the differential in gel electrophoresis (DIGE) technique (8). Specifically, the iTRAQ method has been used to study a variety of diseases and has been shown to be effective and accurate $(4,5,9)$.

The purpose of this study was to analyze serum protein levels using iTRAQ in normal controls, as well as patients with chronic hepatitis $\mathrm{B}(\mathrm{CHB})$ and $\mathrm{HBV}$-induced ACLF, and to verify those results using western blotting. The ultimate aim was to identify the differences in serum protein levels that were closely associated with the progression of hepatitis B and HBV-induced ACLF. The results of this study may provide crucial information regarding viral mechanisms and the pathogenic process.

\section{Materials and methods}

Patients and specimens. Serum samples from healthy individuals and patients with $\mathrm{CHB}$ and $\mathrm{HBV}$-induced ACLF were obtained from the Department of Infectious Diseases and the Department of Traditional Chinese Medicine of the Third Affiliated Hospital of Sun Yat-sen University (Guangzhou, 


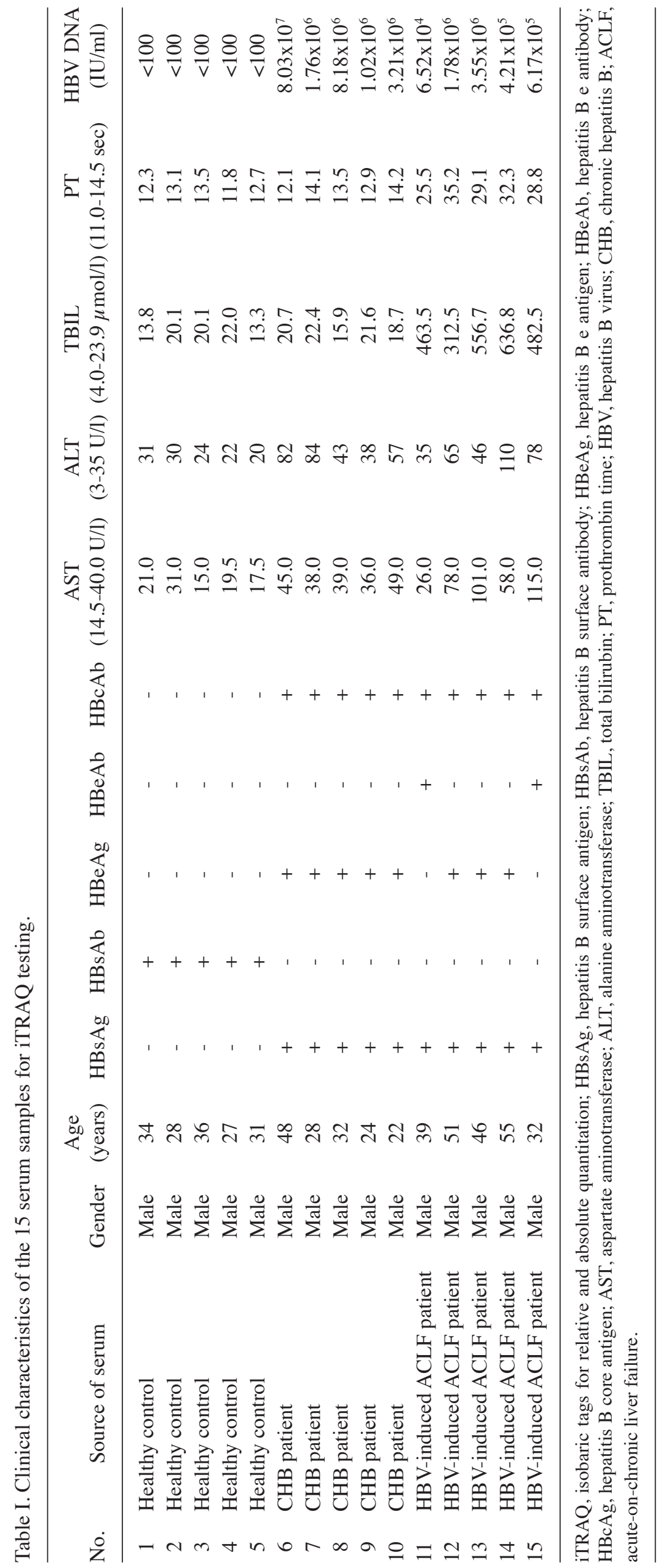


China) (Tables I and II). HBV-induced ACLF was diagnosed using the previously described criteria $(10,11)$. The exclusion criteria included pregnant and lactating females, patients that had been treated with antivirals or immunomodulatory therapy within the previous six months, the presence of other factors causing active liver diseases (e.g. autoimmune, drug-induced liver, alcoholic liver and inherited metabolic liver diseases), concomitant human immunodeficiency virus (HIV) infection or congenital immune deficiency diseases, liver cancer or other malignancies, severe diabetes, autoimmune diseases, other important organ dysfunctions (e.g. kidney dysfunction), concomitant infection (e.g. fever, leukocytosis or neutrophilia; manifestations of abdominal, biliary tract or lung infection) or other serious comorbidities (e.g. hepatic encephalopathy and gastrointestinal bleeding).

The study protocolconformed totheEthical Guidelines of the 1975Declaration of Helsinkiand wasapproved by the appropriate Institutional Review Committee of Third Affiliated Hospital of Sun Yat-sen University (Guangzhou, China) and the Bureau of Health (Guangdong, China). Informed patient consent was obtained prior to participation in this study.

Sample preparation and protein extraction. Plasma was fractionated withProteinMiner Protein Enrichment Small-Capacity kit, Cat\# 163-3006, (ProteinMiner ; Bio-Rad Laboratories, Hercules, CA, USA) according to the manufacturer's instructions. Protein solutions were reduced for $1 \mathrm{~h}$ at $56^{\circ} \mathrm{C}$ with $10 \mathrm{mM}$ dithiothreitol (DTT) and were cysteine-blocked with $55 \mathrm{mM}$ iodoacetamide (IAA) at room temperature for $10 \mathrm{~min}$. Each sample was precipitated with four-times the volume of cold acetone. The total protein concentration was determined using the Bradford assay.

Protein digestion and peptide tagging. Protein solutions [100 $\mu \mathrm{g} ; 5 \mathrm{mg} / \mathrm{ml}$ in $0.5 \mathrm{M}$ triethyl ammonium bicarbonate (TEAB) containing $0.1 \%$ sodium dodecyl sulfate, $\mathrm{pH} 8.5$ ]were digested for $24 \mathrm{~h}$ with $10 \mu \mathrm{g} \mathrm{L}$-1-(4-tosylamido)-2-phenylethyl tosylphenylalanyl chloromethyl ketone (TPCK)-treated trypsin. Each peptide solution was labeled for $3 \mathrm{~h}$ at room temperature using an iTRAQ reagent that had been reconstituted in 70 liters of ethanol, in accordance with the iTRAQ Reagents Multiplex kit instructions (Applied Biosystems, Foster City, CA, USA). The reaction was terminated by adding MilliQ water, and the samples were labeled with $114,115,116$ and 117 mass-tagged iTRAQ reagents.

Strong cation exchange chromatography (SCX). SCX was performed to remove excess iTRAQ reagents and interfering substances for the mass analysis. The labeled peptides were then dried in a vacuum centrifuge and resuspended in $200 \mathrm{ml}$ Buffer A, prior to being loaded on a Phenomenex Luna $55 \mu \mathrm{m}$ SCX 100A column [250x4.60 mm (length $\mathrm{x}$ internal diameter), $5 \mu \mathrm{m}$; Phenomenex, Inc., Torrance, CA, USA] on an Agilent 1100 HPLC unit (Agilent Technologies, Santa Clara, CA, USA). Buffer A consisted of $10 \mathrm{mM} \mathrm{KH}_{2} \mathrm{PO}_{4}$ and $25 \%$ acetonitrile at $\mathrm{pH} 3.0$, while Buffer B consisted of $10 \mathrm{mM}$ $\mathrm{KH}_{2} \mathrm{PO}_{4}, 25 \%$ acetonitrile and $2 \mathrm{M} \mathrm{KCl}$ at $\mathrm{pH} 3.0$. The $60 \mathrm{~min}$ gradient comprised $100 \%$ Buffer A for 30 min, 0-5\% Buffer B for $1 \mathrm{~min}, 5-30 \%$ Buffer B for $15 \mathrm{~min}, 30-50 \%$ Buffer B for 5 min, 50\% Buffer B for 4 min and 50-100\% Buffer A for 


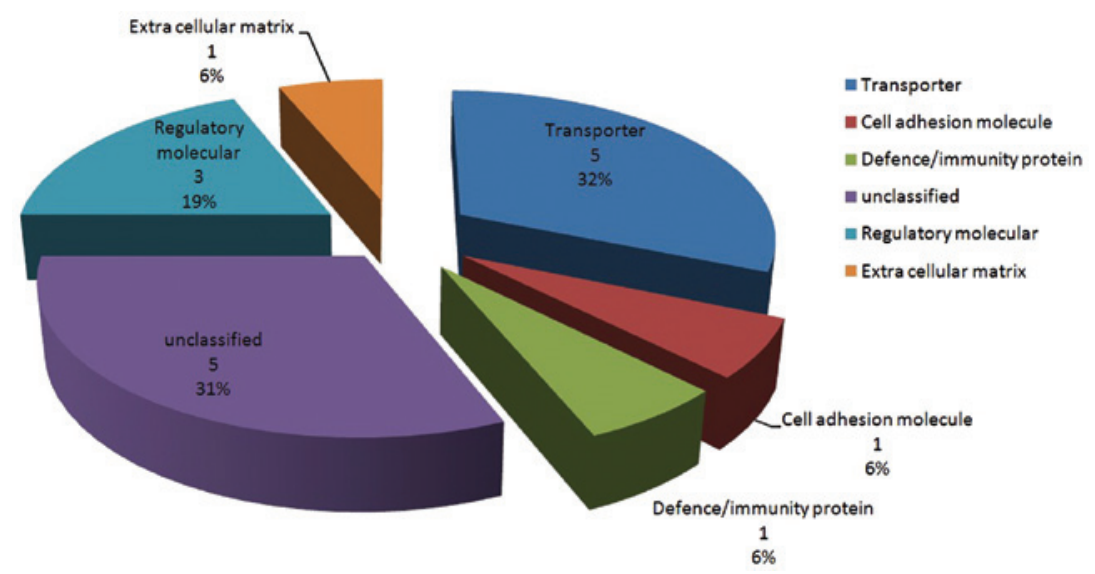

Figure 1. Classification of 16 serum proteins into different categories, based on protein function. The six categories were transporter, cell adhesion molecule, defence/immunity protein, regulatory molecular, extracellular matrix and unclassified.

5 min. Thirteen fractions were collected using a Foxy JR Fraction Collector (Dionex Corp., Sunnyvale, CA, USA). Fractions 2 and 3 were pooled according to the chromatogram profile, based on the peak intensity. All fractions were then dried in a vacuum concentrator and stored at $-20^{\circ} \mathrm{C}$, prior to further analysis using mass spectrometry.

Nano liquid chromatography (LC) coupled to quadrupole time-of-flight ( $Q$-TOF) with tandem mass spectrometry $(M S / M S)$. Peptides from the SCX fractions were dissolved in $0.3 \mu 1100 \%$ formic acid and diluted to $5 \mu 1$ in $0.05 \%$ trifluoroacetic acid (TFA). The peptides were loaded onto a micrOTOF-Q II-nano LC system (Bruker Daltonics, Inc., Billerica, MA, USA) and mass spectra were acquired in the $250-1,600 \mathrm{~m} / \mathrm{z}$ range every second for $60 \mathrm{~min}$.

Database screening. Data were processed using BioTools software (Bruker Daltonics, Inc.). The files were subsequently submitted to an in-house Mascot server (Matrix Science Ltd., London, UK) for database screening. The data were screened against the human sequence database [National Center for Biotechnology Information non-redundant (NCBInr)]. The search was performed using trypsin as a specific enzyme. A maximum of one missed cleavage was permitted and oxidation (M), iTRAQ 4 plex (K) and iTRAQ 4 plex (N-term) were selected as variable modifications. The data obtained on the micrOTOF-Q were searched with a peptide mass tolerance of $10 \mathrm{ppm}$ and a fragment mass tolerance of $0.8 \mathrm{Da}$. Protein ratios were normalized using the overall median ratio for all the peptides in the sample for each separate ratio in every individual experiment. The ratio for a given protein was calculated by taking the average of all the peptide ratios that identified the protein. The final list of protein ratios was an average of the protein ratios of the three experiments and consisted only of proteins discovered in $\geq 2$ of the three experiments. Function definitions of the variable protein contents were searched for using the following two websites: http://www.uniprot.org/ and http://www.ncbi.nlm.nih.gov/.

Western blotting. Five proteins [C-reactive protein (CRP) precursor, hemoglobin $\beta$ chain variant $\mathrm{Hb} \mathrm{S}$-Wake, apolipoprotein $\mathrm{J}$ precursor, platelet factor 4 precursor and vitronectin
$(\mathrm{VN})]$, which demonstrated the greatest differences in their expression levels and the most significant correlation with liver diseases, were chosen and verified by western blotting using western blotting kits (Forevergen, Guangzhou, China). Briefly, the protein lysates were separated by polyacrylamide gel electrophoresis, transferred to a polyvinylidene difluoride (PVDF) membrane and subjected to immunoblotting with the following antibodies: Anti-apolipoprotein J precursor antibody (ab16077; dilution, 1:2,000; Abcam, Cambridge, UK), anti-hemoglobin $\beta$ (sc-21757; dilution, 1:500; Santa Cruz Biotechnology, Inc., Santa Cruz, CA, USA), anti-CRP precursor (ab32412; dilution, 1:1,000; Abcam), anti-platelet factor 4 precursor (AB1488P; dilution, 1:5,000; Millipore, Billerica, MA, USA) and anti-VN (ab11591; dilution, 1:500; Abcam) at $4^{\circ} \mathrm{C}$ overnight. After washing, the membranes were incubated with horseradish peroxidase-conjugated secondary antibodies and visualized using an enhanced chemiluminescence system (ECL; Forevergen, Guangzhou, China).

\section{Results}

Tables I and II summarize the clinical characteristics of the patients from whom the samples were collected. iTR AQ identified 16 different proteins that had $\geq 1.5$-fold differences in expression level between the patients with HBV-induced ACLF and CHB, respectively, and the healthy controls (Table III). Protein quantification software, based on the relative content of the isotopic reporter group and using m/z 114 as a reference, showed significant results $(\mathrm{P} \leq 0.05)$. Some identified protein was lost in the corresponding reporter group, giving no quantification information. The specific information and re-identification will be published in a future study.

The 16 proteins that were identified using iTRAQ were classified into six categories, based on protein function (Fig. 1). The five proteins that demonstrated the greatest differences in their expression and the most significant correlation with liver diseases were verified using western blotting: CRP precursor, hemoglobin $\beta$ chain variant $\mathrm{Hb}$ S-Wake, apolipoprotein J precursor, platelet factor 4 precursor and VN (Fig. 2 and Table IV). Two of the five proteins were not classified according to biological processes (apolipoprotein J precursor and platelet factor 4 precursor). 


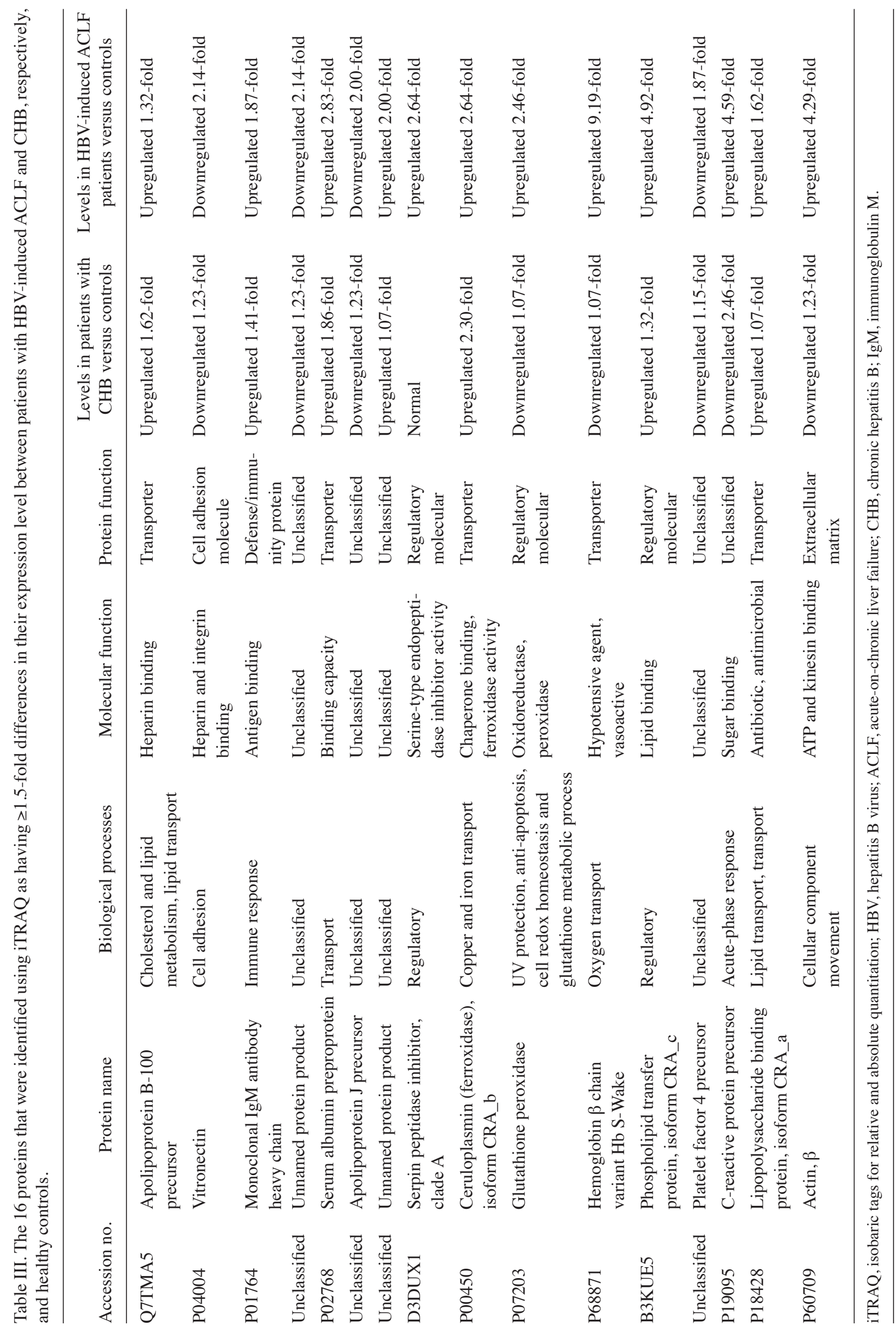




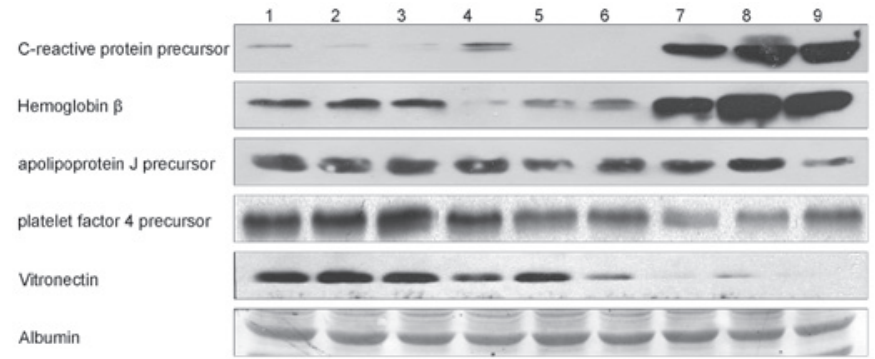

Figure 2. Results of verification using western blotting. Lanes 1-3, normal serum; 4-6, serum from patients with chronic hepatitis B (CHB); 7-9, serum from patients with hepatitis B virus (HBV)-induced acute-on-chronic liver failure (ACLF).

\section{Discussion}

Proteomics research is a potentially useful and effective tool for studying pathogenesis, establishing prognosis and determining treatment outcomes in a variety of diseases. In the field of $\mathrm{CHB}$, proteomics is not widely performed, presumably due to the mechanism of CHB being so complicated. The aim of this study was to describe the changes in serum protein levels in patients with CHB and HBV-induced ACLF, respectively, compared with healthy controls using iTRAQ and western blotting. The authors hypothesized that this approach had the potential to ultimately be beneficial for the identification of proteins that were important in the progression of HBV infections.

In the present study, 16 unique proteins were identified in patients with CHB and those with HBV-induced ACLF that had $\geq 1$.5-fold differences in expression compared with those in the healthy controls. Those proteins belonged to six different categories based on protein function, while two out of the five proteins that were analyzed using western blotting were not classified according to biological processes (apolipoprotein J precursor and platelet factor 4 precursor). The biological functions of the remaining proteins in HBV infection and progression that were analyzed have not yet been elucidated; however, a number of theories are discussed in the following section.

CRP, synthesized in the liver, is an acute phase protein that is rapidly identified in the plasma in cases of infection or tissue damage. CRP is able to activate complement and enhance phagocytosis to facilitate the removal of pathogenic microorganisms and injured, necrotic and apoptotic cells. Therefore, CRP is important in natural immunity $(12,13)$. Originally, CRP was considered to be a nonspecific biomarker of inflammation; however, following $\sim 10$ years of investigation, it has been demonstrated that CRP participates in inflammation and atherosclerosis directly and has been indicated to be a prognostic factor and a risk factor (14). In this study, it was revealed that levels of the precursor of CRP were decreased in the patients with CHB and markedly increased in the patients with ACLF. Nearly identical changes were observed for the hemoglobin $\beta$ chain variant $\mathrm{Hb}$ S-Wake, which has been implicated in in orphan diseases (15), although not in liver diseases.

The apolipoprotein $\mathrm{J}$ precursor is important in cell aging and tumorigenesis with apolipoprotein J, lipid transfer inhibitor protein, complement $\mathrm{C} 3 \mathrm{~d}$, corticosteroid-binding globulin and 
apolipoprotein L1 $(16,17)$. These latter five markers of fibrosis are secreted in the blood and show consistent changes with the increasing stage of fibrosis when compared with the markers used in the FibroTest and enhanced liver fibrosis (ELF), Hepascore and FIBROSpect tests. In the present study, the levels of apolipoprotein $\mathrm{J}$ precursor decreased in the progression of HBV from CHB to ACLF. Therefore, further studies, with a larger number of samples, are required in order to better establish the role of apolipoprotein $\mathrm{J}$ precursor as a potential biomarker for HBV progression.

Platelet factor 4 is chemotactic for numerous cell types. It also functions as an inhibitor of hematopoiesis, angiogenesis and T-cell function, which may be used as a biomarker for establishing a prognosis in severe acute respiratory syndrome (18). Changes in platelet morphology, with accompanying increases in megathrombocyte fraction, may occur in chronic liver diseases, and thrombocytes are activated in chronic liver diseases and liver cirrhosis. Platelet sensitivity to stimuli in patients with liver cirrhosis has been demonstrated to be higher than in healthy controls (19) and it was indicated that platelet factor 4 was activated and upregulated in chronic hepatitis and liver cirrhosis. In the present study, levels of platelet factor 4 precursor decreased in the progression of hepatitis B to liver failure. This result suggests that further studies are required to investigate the potential of platelet factor 4 precursor as a biomarker in the progression of $\mathrm{CHB}$.

$\mathrm{VN}$ is another protein that commonly features in studies on liver disease. It is a multifunctional plasma glycoprotein produced by hepatocytes that is detectable in plasma and the extracellular matrix. Glycosylation of $\mathrm{VN}$ modulates multimerization and collagen binding in liver regeneration (20) and alterations in VN glycosylation modulate substrate adhesion to rat hepatic stellate cells (HSCs), which is responsible for matrix restructuring (21). Immunoblotting data identified increases in VN levels in cirrhotic livers, and VN immunoreactivity has been shown to be markedly increased in the cirrhotic liver matrix, irrespective of the apparent reduction in plasma $\mathrm{VN}$. VN immunoreactivity in the liver may be considered to be a marker of fibrosis, particularly for chronic/mature fibrosis, paralleling previous observations concerning the enhanced orcein staining of cirrhotic septa (22). These results suggest that VN may be important in the progression of liver disease and/or in hepatic fibrosis through its collagen-binding domain I, and that VN levels are likely to be higher in patients with chronic hepatitis, liver cirrhosis and liver cancer than in controls (20-23). In the present study, $\mathrm{VN}$ levels decreased in the progression of hepatitis B (from CHB to HBV-induced ACLF). The observation of decreased $\mathrm{VN}$ levels was consistent with two other studies $(24,25)$. In one of these studies, it was demonstrated that, in chronic liver disease, the concentration of plasma VN was significantly lower than in healthy controls and was associated with the severity of liver disease. Notably, levels of $\mathrm{VN}$ in the liver tissue were significantly increased in patients with chronic liver disease compared with those in normal controls. These results suggested that VN was deposited in injured tissue during the repair process and functioned as an adhesive protein (24). In the other study, it was demonstrated that there were lower levels of plasma $\mathrm{VN}$ in chronic liver disease, which was possibly due to a decreased synthesis, deposition in injured tissues or a combination of the two (25). The same group of authors also revealed that decreased levels of plasma $\mathrm{VN}$ and fibronectin $(\mathrm{FN})$ and increased levels of serum laminin (LM) P1 in patients with chronic liver diseases were associated with hepatic dysfunction, and that changes in the levels of the glycoproteins involved in cell attachment were significant in the development of hepatic fibrosis in patients with chronic liver diseases (25). Based on the accumulating information on $\mathrm{VN}$ and liver fibrosis, it appears that $\mathrm{VN}$ requires further study.

In the present study, the authors aimed to describe changes in serum protein levels in patients with liver dysfunction induced by hepatitis $\mathrm{B}$ and patients with severe liver damage (ACLF). The purpose of this was to specifically identify one or more proteins that are able serve as biomarkers for predicting a pathogenic condition and for prognostic purposes, similar to $\alpha$-fetoprotein (AFP) for hepatocellular carcinoma (HCC). It was not possible in this study to obtain a series of serum samples from individual patients as they progressed through the various stages of hepatitis B infection to severe liver damage (ACLF). However, using iTRAQ and western blotting, several candidate proteins were identified (namely apolipoprotein $\mathrm{J}$ precursor, platelet factor 4 precursor and $\mathrm{VN}$ ) that warrant further study using a larger number of samples.

\section{Acknowledgements}

The authors would like to thank Miss. Qin Zhang and Mr. Bing-Quan Lai for their commitment to this study. This study was supported by the National Natural Science Foundation of China (grant no. 81101256), National Science and Technology Major Projects (grant nos. 2012ZX10002004 and 2012ZX10002007), the Fundamental Research Funds for the Central Universities (grant no. 12ykpy35) and the New Teacher Fund of the Ministry of Education (grant no. 20090171120079).

\section{References}

1. Chinese Society of Hepatology and Chinese Society of Infectious Diseases, Chinese Medical Association: The guideline of prevention and treatment for chronic hepatitis B (2010 version). Zhonghua Gan Zang Bing Za Zhi 19: 13-24, 2011 (In Chinese).

2. Lu FM and Zhuang H: Management of hepatitis B in China. Chin Med J (Engl) 122: 3-4, 2009.

3. Ren F, Chen Y, Wang Y, Yan Y, Zhao J, Ding M, Zhang J, Jiang Y, Zhai Y and Duan Z: Comparative serum proteomic analysis of patients with acute-on-chronic liver failure: alpha-1-acid glycoprotein maybe a candidate marker for prognosis of hepatitis B virus infection. J Viral Hepat 17: 816-824, 2010.

4. Yang L, Rudser KD, Higgins L, Rosen HR, Zaman A, Corless CL, David L and Gourley GR: Novel biomarker candidates to predict hepatic fibrosis in hepatitis C identified by serum proteomics. Dig Dis Sci 56: 3305-3315, 2011.

5. Jin GZ, Li Y, Cong WM, Yu H, Dong H, Shu H, Liu XH, Yan GQ, Zhang L, Zhang Y, et al: iTRAQ-2DLC-ESI-MS/MS based identification of a new set of immunohistochemical biomarkers for classification of dysplastic nodules and small hepatocellular carcinoma. J Proteome Res 10: 3418-3428, 2011.

6. Lee HJ, Na K, Choi EY, Kim KS, Kim H and Paik YK: Simple method for quantitative analysis of N-linked glycoproteins in hepatocellular carcinoma specimens. J Proteome Res 9: 308-318, 2010.

7. Goh WW, Lee YH, Zubaidah RM, Jin J, Dong D, Lin Q Chung MC and Wong L: Network-based pipeline for analyzing MS data: an application toward liver cancer. J Proteome Res 10: 2261-2272, 2011. 
8. Wu WW, Wang G, Baek SJ and Shen RF: Comparative study of three proteomic quantitative methods, DIGE, cICAT, and iTRAQ, using 2D gel- or LC-MALDI TOF/TOF. J Proteome Res 5: 651-658, 2006.

9. Kolla V, Jenö P, Moes S, Tercanli S, Lapaire O, Choolani M and Hahn S: Quantitative proteomics analysis of maternal plasma in Down syndrome pregnancies using isobaric tagging reagent (iTRAQ). J Biomed Biotechnol 2010: 952047, 2010.

10. Lok AS and McMahon BJ: Chronic hepatitis B: update 2009. Hepatology 50: 661-662, 2009.

11. Sarin SK, Kumar A, Almeida JA, Chawla YK, Fan ST, Garg H, et al: Acute-on-chronic liver failure: consensus recommendations of the Asian Pacific Association for the Study of the Liver (APASL). Hepatol Int 3: 269-282, 2009.

12. Nahum E, Livni G, Schiller O, Bitan S, Ashkenazi S and Dagan O: Role of C-reactive protein velocity in the diagnosis of early bacterial infections in children after cardiac surgery. J Intensive Care Med 27: 191-196, 2012.

13. Sklavou R, Karavanaki K, Critselis E, Kossiva L, Giannaki M, Tsolia M, Papadakis V, Papargyri S, Vlachou A, Karantonis F, Gourgiotis D and Polychronopoulou S: Variation of serum $\mathrm{C}$-reactive protein (CRP) over time in pediatric cancer patients with febrile illness and its relevance to identified pathogen. Clin Biochem 45: 1178-1182, 2012.

14. Kashiwagi M, Tanaka A, Kitabata H, Tsujioka H, Matsumoto $H$, Arita Y, Ookochi K, Kuroi A, Kataiwa H, Tanimoto T, et al: Relationship between coronary arterial remodeling, fibrous cap thickness and high-sensitivity C-reactive protein levels in patients with acute coronary syndrome. Circ J 73: 1291-1295, 2009.

15. Kutlar F, Redding-Lallinger R, Meiler SE, Bakanay SM, Borders $\mathrm{L}$ and Kutlar A: A new sickling variant 'Hb S-Wake $\beta$ [(Glu6Val-Asn139 Ser)]' found in a compound heterozygote with $\mathrm{Hb} \mathrm{S} \beta$ (Glu6Val) coinherited with homozygous $\alpha$-thalassemia-2: phenotype and molecular characteristics. Acta Haematol 124: $120-124,2010$

16. Trougakos IP and Gonos ES: Clusterin/apolipoprotein J in human aging and cancer. Int J Biochem Cell Biol 34: 1430-1448, 2002.
17. Gangadharan B, Bapat M, Rossa J, Antrobus R, Chittenden D, Kampa B, Barnes E, Klenerman P, Dwek RA and Zitzmann N: Discovery of novel biomarker candidates for liver fibrosis in hepatitis C patients: a preliminary study. PLoS One 7: e39603, 2012.

18. Poon TC, Pang RT, Chan KC, Lee NL, Chiu RW, Tong YK, Chim SS, Ngai SM, Sung JJ and Lo YM: Proteomic analysis reveals platelet factor 4 and beta-thromboglobulin as prognostic markers in severe acute respiratory syndrome. Electrophoresis 33: 1894-1900, 2012.

19. Panasiuk A, Prokopowicz D, Zak J, Matowicka-Karna J, Osada J and Wysocka J: Activation of blood platelets in chronic hepatitis and liver cirrhosis P-selectin expression on blood platelets and secretory activity of beta-thromboglobulin and platelet factor- 4 . Hepatogastroenterology 48: 818-822, 2001.

20. Sano K, Asanuma-Date K, Arisaka F, Hattori S and Ogawa H: Changes in glycosylation of vitronectin modulate multimerization and collagen binding during liver regeneration. Glycobiology 17: 784-794, 2007.

21. Sano K, Miyamoto Y, Kawasaki N, Hashii N, Itoh S, Murase M, Date K, Yokoyama M, Sato C, Kitajima K and Ogawa H: Survival signals of hepatic stellate cells in liver regeneration are regulated by glycosylation changes in rat vitronectin, especially decreased sialylation. J Biol Chem 285: 17301-17309, 2010.

22. Koukoulis GK, Shen J, Virtanen I and Gould VE: Vitronectin in the cirrhotic liver: an immunomarker of mature fibrosis. Hum Pathol 32: 1356-1362, 2001.

23. Yamada S, Kobayashi J, Murawaki Y, Suou T and Kawasaki H: Collagen-binding activity of plasma vitronectin in chronic liver disease. Clin Chim Acta 252: 95-103, 1996.

24. Kobayashi J, Yamada S and Kawasaki H: Distribution of vitronectin in plasma and liver tissue: relationship to chronic liver disease. Hepatology 20: 1412-1417, 1994.

25. Tomihira M: Changes in plasma vitronectin, fibronectin, and serum laminin P1 levels and immunohistochemical study of vitronectin in the liver of patients with chronic liver diseases. Fukuoka Igaku Zasshi 82: 21-30, 1991 (In Japanese). 